\title{
The reason for existence of discrete breathers in 2D and 3D Morse crystals
}

\author{
E.A. Korznikova ${ }^{1}$, A.A. Kistanov ${ }^{1, \dagger}$, K.S. Sergeev ${ }^{2}$, D.A. Shepelev², A.R. Davletshin ${ }^{3}$, \\ D.I. Bokij ${ }^{4}$ S.V. Dmitriev ${ }^{1,5}$ \\ †andrei.kistanov.ufa@gmail.com
}

${ }^{1}$ Institute for Metals Superplasticity Problems RAS, 39 Khalturin Str., Ufa, 450001, Russia

${ }^{2}$ Saratov State University, 83 Astrakhanskaya Street, Saratov, 410012

${ }^{3}$ Ltd. BashNIPIneft, 30/1 Karl Marx St., Ufa, 450077, Russia

${ }^{4}$ ATOMPROEKT 82, Savushkina St., Saint Petersburg, 197183, Russia

${ }^{5}$ National Research Tomsk State University, Lenin Ave, 36, Tomsk 634050, Russia

It is known that crystals can support discrete breathers (DBs) - periodic in time and spatially localized vibrational modes. $\mathrm{DB}$ does not radiate energy, as its frequency does not lie within the spectrum of small-amplitude traveling waves (phonons). DB frequency can leave the spectrum of low-amplitude oscillations due to the nonlinearity of the interatomic potentials, as it is well known that the frequency of a nonlinear oscillator depends on the amplitude. Theoretically, it was shown that DB cannot exist in a one-dimensional chain of identical point masses interacting with each other through the Toda, Born-Mayer, Lennard-Jones or Morse potential. The reason of non-existence of DB is the softness of the considered potentials, which does not allow to form a spatially localized mode with frequency above the phonon spectrum. On the basis of this rigorous result, it was concluded that because of the softness of the interatomic interactions in crystals with a simple structure (e.g., in pure metals) existence of DB is very unlikely. Attention should be paid to crystals with a gap in phonon spectrum. In such crystals localized vibrational modes may have frequencies decreasing with amplitude and entering the gap of the phonon spectrum. The first successful attempt to excite a gap DB in alkali halide NaI crystal dates back to 1997, for this purpose, the method of molecular dynamics was used. However, in 2011 DBs with frequencies higher than the phonon spectrum were discovered in pure metals, which poses the question about the conditions of the existence of DBs in crystals with realistic interatomic potentials. In this paper we show that the dimension of the crystal is important, and the Morse crystals of dimension higher than one can support DBs with frequencies above the phonon spectrum.

Keywords: discrete breather, intrinsic localized mode, Morse potential, nonlinear lattice dynamics, molecular dynamics.

\section{Почему существуют дискретные бризеры в двумерных и трехмерных моноатомных кристаллах Морзе?}

\author{
Корзникова Е.А. ${ }^{1}$, Кистанов А.А. ${ }^{1, \dagger}$, Сергеев К.С. ${ }^{2}$, Шепелев И.А. ${ }^{2}$, Давлетшин А.Р. ${ }^{3}$, \\ Бокий Д.И. ${ }^{4}$ Дмитриев С.В. ${ }^{1,5}$ \\ †andrei.kistanov.ufa@gmail.com
}

\begin{abstract}
${ }^{1}$ Институт проблем сверхпластичности металлов РАН, ул. Халтурина 39, 450001, Уфа, Россия
${ }^{2}$ Саратовский государственный университет имени Н.Г. Чернышевского, ул. Астраханская 83, 410012, Саратов, Россия

${ }^{3}$ ООО БашНИПИнефть, ул. Карла Маркса 30/1, 450077, Уфа, Россия

${ }^{4}$ АО "АТОМПРОЕКТ", ул. Савушкина 82, 197183, Санкт-Петербург, Россия

${ }^{5}$ Национальный исследовательский Томский государственный университет, просп. Ленина 36, 634050 Томск, Россия

Известно, что кристаллы могут поддерживать существование дискретных бризеров (ДБ) - периодических во времени пространственно-локализованных колебательных мод. ДБ не излучают энергию, так как их частота не лежит в спектре малоамплитудных бегущих волн (фононов). Выход частоты ДБ из спектра малоамплитудных колебаний происходит за счет нелинейности во взаимодействии атомов, ведь известно, что частота нелинейных осцилляторов зависит от амплитуды колебаний. Теоретически было показано, что ДБ не могут существовать в одномерной цепочке одинаковых точечных масс, взаимодействующих друг с другом посредством потенциалов Тоды, Борн-Маера,
\end{abstract}


Леннарда-Джонса или Морзе. В качестве причины была названа мягкость данных потенциалов, которая не позволяла сформировать пространственно-локализованную моду с частотой выше спектра фононных колебаний. На основании данного строгого результата был сделан вывод о том, что из-за мягкости межатомных взаимодействий в кристаллах с простой структурой (например, в чистых металлах) ДБ, вероятнее всего, не существуют, и при поиске ДБ следует обратить внимание на кристаллы со щелью в фононном спектре. В таких кристаллах локализованная колебательная мода может иметь частоты, уменьшающиеся с амплитудой колебаний и входящие в щель фононного спектра. Первая успешная попытка возбуждения щелевого ДБ в щелочно-галоидном кристалле NaI датируется 1997 годом, для этой цели использовался метод молекулярной динамики. Однако в 2011 году ДБ с частотами выше фононного спектра были обнаружены в чистых металлах, что заставило задуматься об условиях существования ДБ в кристаллах с реалистичными межатомными потенциалами. В настоящей работе показано, что размерность кристалла имеет важное значение, и в Морзевских кристаллах размерности выше единицы ДБ существовать могут.

Ключевые слова: дискретный бризер, потенциал Морзе, нелинейные колебания решетки, молекулярная динамика.

\section{1. Введение}

Дискретными бризерами (ДБ) называются локализованные в пространстве и периодические во времени колебательные моды в бездефектных нелинейных решетках. В ряде работ была показана возможность существования ДБ в кристаллах [1-5], что привело к всплеску научного интереса к изучению ДБ и их свойств в кристаллах различного типа [6-16]. В настоящее время концепция ДБ все чаще привлекается для объяснения различных физических эффектов в твердых телах [17-21].

ДБ не излучают свою энергию в виде малоамплитудных колебаний (фононов), поскольку их частоты лежат вне спектра фононных колебаний кристалла. Следовательно, при изучении ДБ необходимо обратить внимание на два основных вопроса. Во-первых, какова структура фононного спектра рассматриваемого кристалла, а именно, наличие/отсутствие щели в фононном спектре. Во-вторых, каким образом частота ДБ может выйти из фононного спектра кристалла. В простых структурах, таких как чистые металлы, щель в фононном спектре отсутствует, что допускает наличие ДБ только с частотами выше фононного спектра кристалла. С другой стороны, кристаллы со сложной структурой могут иметь щель в фононном спектре, в этом случае возможно появление щелевых ДБ, то есть, ДБ с частотами колебаний в щели фононного спектра. Стоит отметить, что частоты ДБ выходят из фононного спектра из-за наличия ангармонизмов межатомных сил, приводящих к зависимости частоты колебаний атомов от амплитуды. ДБ может демонстрировать два типа нелинейности - жесткий и мягкий, при которых частота моды, соответственно, увеличивается или уменьшается с ростом амплитуды. Очевидно, что ДБ с мягким типом нелинейности возможны только в кристаллах со щелью в фононном спектре. В этом случае частота ДБ отщепляется от верхнего края щели и, уменьшаясь с ростом амплитуды, входить в щель фононного спектра. Частоты щелевых ДБ с жестким типом нелинейности отходят от нижней границы щели фононного спектра и растут с увеличением амплитуды ДБ. В кристаллах со сплошным спектром возможно наличие ДБ только с жестким типом нелинейности, когда частота ДБ растет с амплитудой, отделяясь от верхнего края фононного спектра.

\section{2. О типе нелинейности класси- ческих парных потенциалов}

Возникает естественный вопрос: какой тип нелинейности, мягкий или жесткий, характерен для межатомных взаимодействий в кристаллических решетках? Отправной точкой в обсуждении данного вопроса стала работа Киселева [6], в которой были рассмотрены одномерные (1D) моноатомные и двухатомные кристаллы (см. рис. 1a, 1b), где атомы взаимодействовали друг с другом посредством одного из пяти парных межатомных потенциалов: полиномиального потенциала $K_{2}-K_{3}-K_{4}$ и потенциалов Тоды, Борн-Маера, Леннарда-Джонса и Морзе. Необходимо отметить, что полиномиальный потенциал и потенциал Тоды дают силу межатомных взаимодействий, которая не стремится к нулю с увеличение межатомного расстояния, и, следовательно, данные потенциалы не могут быть использованы для учета дальних взаимодействий. Три другие из рассматриваемых потенциалов пригодны для этих
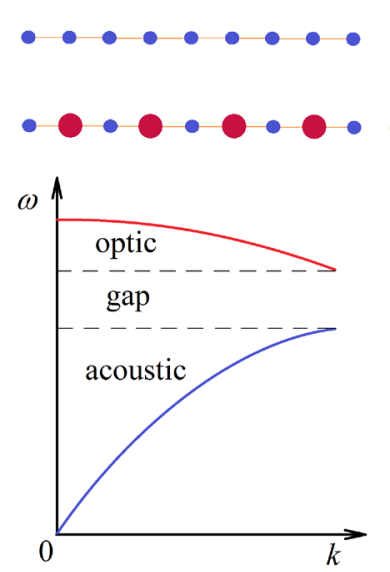

Рис. 1. Одномерная модель (a) моноатомного и (b) двухатомного кристаллов. (c) Схематическая картина фононных дисперсионных кривых для одномерного биатомного кристалла. Акустическая и оптическая ветви разделены щелью. Спектр моноатомного кристалла является бесщелевым ввиду отсутствия оптической составляющей.

Fig. 1. One-dimensional model of (a) monatomic and (b) biatomic crystals. (c) Schematic picture of the pho-non dispersion curves for the biatomic crystal. Acoustic and optic bands are separated by a gap. Spectrum of monatomic crystal does not possess an optic band and thus it is gapless. 
целей и широко используются в молекулярно-динамических расчетах. Основной вывод работы [6] заключается в том, что реалистичные потенциалы Борн-Маера, Леннарда-Джонса и Морзе создают мягкий тип нелинейности и не поддерживают наличия локализованных мод с частотами выше фононного спектра. В двухатомном кристалле с наличием щели в фононном спектре (см. рис. 1с) авторам удалось возбудить только щелевые ДБ с мягким типом нелинейности. Из чего было сделано заключение, что типичные межатомные потенциалы поддерживают наличие мод лишь с мягким типом нелинейности, и маловероятно обнаружить ДБ в кристаллах со сплошным спектром, например, в чистых металлах [6]. В связи с этим исследователи обратили интерес к кристаллам, предполагающим наличие щели в фононном спектре [6]. Первая успешная попытка в моделировании ДБ была осуществлена для щелочно-галоидного кристалла $\mathrm{NaI}$ с ионным межатомным взаимодействием [7]. Для этого же кристалла, была проведена серия экспериментов по обнаружению ДБ при высоких температурах в тепловом равновесии [22].

Однако в действительности, все реалистичные потенциалы имеют точку перегиба, где вторая производная меняет знак с положительного на отрицательный с увеличением межатомного расстояния. Таким образом, потенциалы являются жесткими при малых и мягкими при больших межатомных расстояниях. Тот факт, что реалистичные межатомные потенциалы имеют твердое ядро и мягкий хвост соотносится с представлением о структуре атомов, имеющих компактное ядро и мягкие электронные облака. На больших расстояниях атомы взаимодействуют в основном посредством электронов, в то время как на малых расстояниях более существенную роль играют взаимодействия ядер атомов. Можно ожидать, что если больший вклад в динамику определенной колебательной моды вносит мягкий хвост потенциала, то частота моды будет уменьшаться с увеличением амплитуды, и будет происходить обратное, если больший вклад вносит жесткое ядро потенциала.

Данный факт легко продемонстрировать, рассмотрев одномерную цепочку атомов единичной массы, где каждый атом взаимодействует с ближайшими соседями посредством потенциала $U(r)$, где $U-$ потенциальная энергия а $r$ - расстояние между парой атомов. Пусть $x_{n}(t)-$ неизвестная координата $n$-ого атома, зависящая от времени $t$. Тогда динамика цепочки описывается следующими уравнениями движения:

$$
\frac{d^{2} x_{n}}{d t^{2}}=U^{\prime}\left(x_{n+1}-x_{n}\right)-U^{\prime}\left(x_{n}-x_{n-1}\right) .
$$

Межатомные взаимодействия опишем потенциалом Морзе, наиболее мягким среди всех рассмотренных в работе [6],

$$
U(r)=D\left(e^{-2 \alpha\left(r-r_{m}\right)}-2 e^{-\alpha\left(r-r_{m}\right)}\right),
$$

где $D, \alpha$ и $r_{m}$ - параметры потенциала. Функция $U(r)$ имеет минимум в точке $r=r_{m}, D-$ энергия взаимодействия (глубина потенциала), $\alpha$ определяет жесткость связи. Нами были выбраны следующие параметры потенциала $D=1, r_{m}=1$ и $\alpha=5$.
В рамках данной модели рассматривается два варианта колебаний моды, показанной на рис. 2а (все атомы имеют одинаковую амплитуду колебаний $A$, при этом каждый атом колеблется в противофазе со своими ближайшими соседями). В первом случае межатомное расстояние $h$ является функцией амплитуды $A$, таким образом, что рассматриваемая система атомов находится под нулевым внешним давлением. Во втором случае расстояние между центрами колебаний атомов неизменно, $h$ $=1$, но при $A>0$ система находится под положительным давлением $p$. Для первого случая на рис. $2 \mathrm{~b}$ представлены зависимости частоты колебательной моды $\omega$ и межатомного расстояние $h$ от амплитуды $A$. Видно, что частота $\omega$ уменьшается с увеличением амплитуды $A$, таким образом, рассматриваемая мода демонстрирует мягкий тип нелинейности. Однако, во втором случае (см. рис. 2c), частота $\omega$ (и давление $p$ ) увеличиваются с увеличением амплитуды $A$, то есть имеем жесткий тип нелинейности. Это можно объяснить тем, что жесткое ядро потенциала Морзе вносит больший вклад в динамику моды при неизменной длине цепочки, в то время как при нулевом давлении, когда расстояние между центрами колебаний атомов увеличивается, больший вклад дает мягкий хвост потенциала.

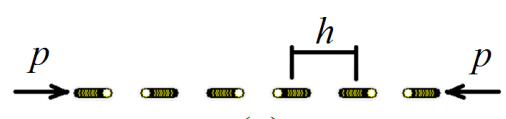

(a)
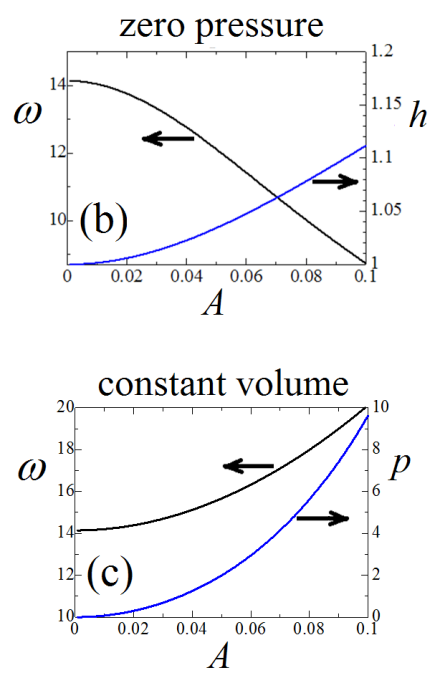

Pис. 2. (a) Колебательная мода в 1D моноатомном кристалле, где все атомы колеблются с одинаковой амплитудой $A$, каждый в противофазе со своими ближайшими соседями. (b) Частота (левая ордината) и расстояние между центрами колебания соседних атомов (правая ордината), как функции амплитуды для случая нулевого внешнего давления. (с) Частота (левая ордината) и давление (правая ордината), как функции амплитуды для случая постоянной длины цепочки $(h=1)$.

Fig. 2. (a) Staggered vibrational mode in 1D mon-atomic crystal. All atoms oscillate with the same amplitude, $A$, each out-of-phase with the nearest neighbors. (b) Frequency (left ordinate) and distance between vibration centers of neighboring atoms (right ordinate) as the functions of $A$ in the case of zero external pressure. (c) Frequency (left ordinate) and pressure (right ordinate) as the functions of $A$ in the case of constant volume $(h=1)$. 
Из выше изложенного очевидно, что ДБ не существуют в одномерной решётке Морзе. Любые локализованные колебательные моды большой амплитуды приведут к локальному “тепловому расширению” и увеличению расстояний между центрами колебаний атомов. При этом мягкий хвост потенциала будет играть большую роль, чем жесткое ядро, и частота моды не будет увеличиваться с ростом амплитуды и не сможет оказаться выше фононного спектра.

\section{3. Дискретные бризеры в двумерных и трех- мерных моноатомных кристаллах Морзе}

Оказалось, что полученный строгий результат работы [6] применим только для одномерного случая. В двухмерной и терехмерной решетках Морзе существуют различные возможности для подавления «теплового расширения» в центре ДБ и его частота может увеличиваться с увеличением амплитуды, выходя за пределы фононного спектра, что делает ДБ устойчивым и долгоживущим.

На рис. 3 показан один из возможных способов возбуждения ДБ в 2D кристалле Морзе. ДБ возбуждался в плотноупакованном атомном ряду, как показано на рис. За. Очевидно, что атомы в центре ДБ не могут свободно отодвигаться друг от друга, так как атомный ряд, где возбуждён ДБ, находится под воздействием периодического потенциала, индуцированного атомами окружающими атомный ряд. На рис. $3 \mathrm{~b}$ представлена схематическая картина цепочки атомов, испытывающих действие периодического потенциала. Действие этого потенциала затрудняет увеличение расстояний между атомами в ядре ДБ так, что жесткое ядро потенциала Морзе дает больший вклад в динамику ДБ, и его частота растет с амплитудой, находясь выше бесщелевого фононного спектра $[23,24]$.

Оказалось, что возможно получить и другой, высокосимметричный ДБ в 2D моноатомной решетке Морзе [25], как это показано на рис. 4а. Такой ДБ так же имеет частоты выше фононного спектра кристалла, так как “тепловое расширение” ядра ДБ подавляется окружающей его областью кристалла.

Далее приведён расчёт давления окружающей среды на ядро ДБ. Отметим, что с удалением от центра ДБ перемещения атомов становятся малыми, в этом случае можно применить линейную теорию упругости для расчета радиального перемещения $u(R)$ и радиальной $\sigma_{R R}(R)$ и тангенциальной $\sigma_{\theta \theta}(R)$ компонент напряжений как функции расстояния от центра ДБ, $R$ (см. рис. $4 \mathrm{~b})$.

Рассматривая проблему плоского напряженного состояния для упругой среды с модулем Юнга $E$ и коэффициентом Пуассона $v$, и принимая во внимание радиальную симметрию задачи, можно записать уравнение равновесия в терминах радиального перемещения следующим образом [26]

$$
\frac{d^{2} u}{d R^{2}}+\frac{1}{R} \frac{d u}{d R}-\frac{u}{R^{2}}=0 .
$$
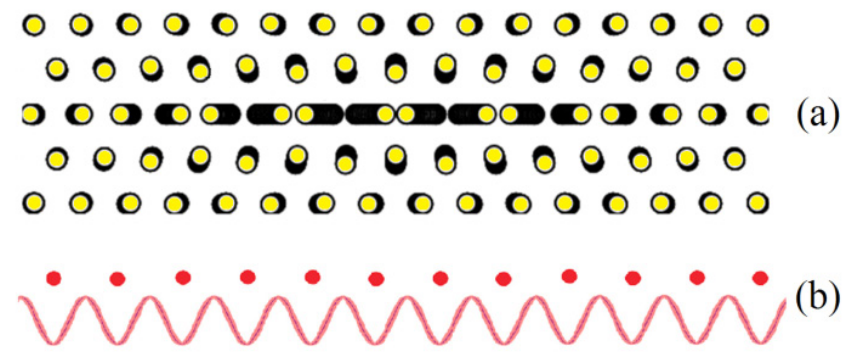

Pис. 3. (а) ДБ в 2D моноатомной решетке Морзе [23]. Десяток атомов одного плотноупакованного ряда колеблются в противофазе со своими ближайшими соседями. “Тепловое расширение" в центре ДБ подавляется периодическим потенциалом, созданным остальной частью кристалла. (b) Схематическая картина цепочки атомов под действием периодического потенциала.

Fig. 3. (a) DB in $2 \mathrm{D}$ monatomic Morse lattice [23]. A dozen of atoms in one close-packed atomic row oscillate out-of-phase with the nearest neighbors. "Thermal expansion" in the core of the DB is suppressed by the periodic on-site potential induced by the rest of the crystal. (b) Schematic picture of the atomic chain in the periodic on-site potential.

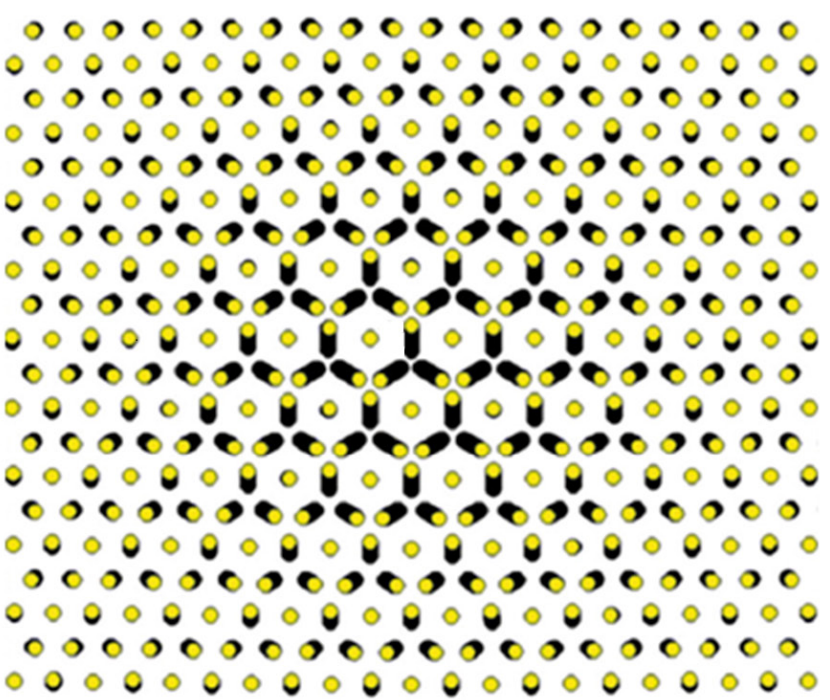

(a)

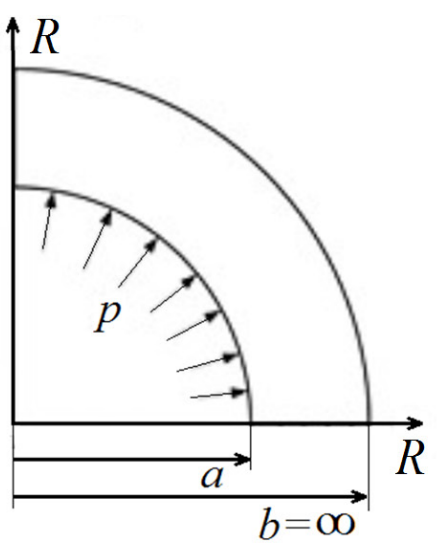

(b)

Pис. 4. Высокосимметричный ДБ в моноатомной 2D решетке Морзе [25].

Fig. 4. High-symmetry DB in monatomic 2D Morse lattice [25]. 
Предположим, что ядро ДБ имеет радиус $а$ и оно оказывает давление $p$ на внешнюю упругую среду. Дальнейшие вычисления представлены для упругого кольца c внешним диаметром $b$. Общее решение уравнения (3) имеет вид

$$
u=C_{1} R+\frac{C_{2}}{R},
$$

где константы интегрирования

$$
C_{1}=\frac{1-v}{E} \frac{a^{2} p}{b^{2}-a^{2}}, \quad C_{2}=\frac{1+v}{E} \frac{a^{2} b^{2} p}{b^{2}-a^{2}},
$$

были выбраны так, чтобы удовлетворить граничным условиям для радиального напряжения на внутреннем и внешнем радиусах кольца, соответственно $\sigma_{R R}(a)=-p$, $\sigma_{R R}(b)=0$. При $b \rightarrow \infty$ имеем $C_{1}=0, C_{2}=a^{2} p(1+v) / E$. Исходя из этого и принимая во внимание выражение (4) находим решение в виде

$$
u=a^{2} p \frac{1+v}{E} \frac{1}{R}, \quad \sigma_{R R}=-\sigma_{\theta \theta}=-p \frac{a^{2}}{R^{2}},
$$

где компоненты напряжений найдены из соотношения между перемещениями и деформациями и закона Гука [26].

Из выражения (6) видно, что ДБ создают дальнодействующие радиальные перемещения, которые медленно затухают, как $R^{-1}$, с увеличением расстояния от центра ДБ. При этом напряжения уменьшаются как $R^{-2}$. Радиальное напряжение сжатия не позволяет ядру ДБ свободно расширяться, что делает вклад жесткого ядра потенциала Морзе достаточно существенным для создания ДБ с жестким типом нелинейности $[23,25]$.

Отметим, что в 3D кристалле при аналогичном рассмотрении ДБ со сферической симметрией и с допущением изотропной упругой среды (заметим, что все 3D кристаллы анизотропны) уравнение равновесия в терминах радиального перемещения можно записать в виде

$$
R \frac{d^{2} u}{d R^{2}}+2 \frac{d u}{d R}-2 \frac{u}{R}=0 .
$$

Решение имеет вид

$$
u=a^{3} p \frac{1+v}{2 E} \frac{1}{R^{2}}, \quad \sigma_{r r}=-2 \sigma_{\theta \theta}=-p \frac{a^{3}}{R^{3}} .
$$

Для 3D случая радиальные перемещения уменьшаются с расстоянием от центра ДБ как $R^{-2}$, а напряжения как $R^{-3}$, что быстрее, чем для $2 \mathrm{D}$ случая. Таким образом, в 3D кристалле Морзе получить ДБ с частотами выше фононного спектра проще, чем в 2D, благодаря более эффективному подавлению «теплового расширения» упругой средой.

Можно заключить, что в 2D и 3D кристаллах с peaлистичными потенциалами взаимодействия с жестким ядром и мягким хвостом, могут существовать ДБ с жестким типом нелинейности. Это возможно благодаря тому, что «тепловое расширение» ядра ДБ подавлено окружающей частью кристалла, при этом жесткое ядро межатомного потенциала вносит больший вклад в динамику ДБ, чем мягкий хвост.

\section{4. Выводы и нерешенные проблемы}

\section{В заключении можно выделить следующее:}

1. Реалистичные потенциалы межатомного взаимодействия имеют жесткое ядро и мягкий хвост, что отражает структуру атомов, имеющих плотное ядро и размытые электронные оболочки. Из-за асимметрии нелинейных потенциалов, при больших амплитудах колебания среднее расстояние между атомами в ядре ДБ увеличивается. Тем не менее, в 2D и 3D кристаллах “термическое расширение” ядра ДБ до некоторой степени подавлено окружающие средой. Поскольку свободное расширение ядра ДБ в 2D и 3D кристаллах невозможно, то жесткое ядро межатомного потенциала вносит больший вклад в динамику ДБ, чем мягкий хвост. В результате, частота ДБ увеличивается с амплитудой и может оказаться выше фононного спектра кристалла.

2. 2D решетка Морзе может поддерживать как минимум два типа ДБ. 3D ГЦК металлы могут поддерживать как минимум три типа ДБ. В упорядоченных сплавах, имеющих щель в фононном спектре кристалла, возможно еще большее разнообразие типов ДБ. Таким образом, возникает задача систематического поиска различных типов ДБ в чистых металлах и упорядоченных сплавах. Не менее важно подробно описать и проанализировать свойства (подвижность, степень локализации, время жизни, диапазон возможных частот, амплитуды и энергии) различных ДБ.

3. Результаты молекулярно-динамических исследований ДБ сильно зависят от типа выбранного межатомного потенциала взаимодействия. Следовательно, важнейшее значение имеют первопринципные (ab initio) расчеты, позволяющие проверить точность результатов молекулярно-динамических расчетов.

4. Дефекты кристаллической решетки играю важную роль в физике кристаллов. В связи с этим важно понять возможную роль ДБ в зарождение или движения дефектов. Для этого, взаимодействие ДБ с дефектами кристаллической решетки и столкновение ДБ друг с другом должны быть детально изучены в различных кристаллах.

5. Нерешенными проблемами для физики кристаллов так же остаются изучение влияние температуры на свойства ДБ и возможность спонтанного возбуждения ДБ в тепловом равновесии при повышенных температурах.

В целом, очень важно найти общие черты и отличительные особенности между ДБ различных типов в кристаллах для того, чтобы искать пути их технологического применения.

Благодарность/Aknowledgements. А.А. К., К.С. C. и И.А. Ш. благодарят грант Российского фонда фундаментальных исследований (РФФИ) № 16-32-00479, работа Е.А. К. поддержана РФФИ в рамках проекта № 16-42-220002 р_a «Физическая природа дискретных бризеров в наноразмерных ГЦК металлах и сплавах» и С.В. Д. благодарит грант Российского научного фонда (РНФ) № 16-12-10175. 


\section{Литература/References}

1. D.K. Campbell, S. Flach, Yu.S. Kivshar. Phys. Today. 57(1), 43(2004).

2. S. Flach, A.V. Gorbach. Phys. Rep. 467, 1, (2008).

3. V. Hizhnyakov, M. Haas, M. Klopov, A. Shelkan. Letters on Materials, 6, 61 (2016).

4. V. Hizhnyakov, M. Haas, A. Shelkan, M. Klopov. Springer Series in Materials Science. 221, 229 (2015).

5. S.V. Dmitriev, E.A. Korznikova, Yu.A. Baimova, M.G. Velarde. Physics-Uspekhi. 59(5), 446 (2016).

6. S.A. Kiselev, S.R. Bickham, A.J. Sievers. Phys. Rev. B. 48, 13508, (1993).

7. S.A. Kiselev, A.J. Sievers. Phys. Rev. B. 55, 5755 (1997).

8. A.A. Kistanov, S.V. Dmitriev. Tech. Phys. Lett. 39, 618 (2013); Pis'ma Zh. Tekh. Fiz. 39(13), 78 (2013).

9. A.A. Kistanov et al. JETP Lett. 99, 353 (2014); Pis'ma Zh. Eksp. Teor. Fiz. 99, 403 (2014).

10. A.A. Kistanov, A.S. Semenov, S.V. Dmitriev. JETP. 119, 766 (2014); Zh. Eksp. Teor. Fiz. 146, 869 (2014).

11. А.А. Кистанов, А.С. Семенов, Р.Т. Мурзаев, С.В. Дмитриев. ФПСМ. 11 (4/2), 572 (2014).

12. А.А. Кистанов. ФПСМ. 11(1), 9 (2014).

13. А.А. Кистанов, А.С. Семенов, Р.Т. Мурзаев, С.В. Дмитриев. ФПСМ. 11 (3), 572 (2014).

14. A.A. Kistanov, S.V. Dmitriev, A.S. Semenov, V.I. Dubinko,
D.A. Terentev. Tech. Phys. Lett. 40, 657 (2014); Pis'ma Zh. Tekh. Fiz. 40(15), 58 (2014).

15. A. A. Kistanov, S.V. Dmitriev, A.P. Chetverikov, M.G. Velarde. Eur. Phys. J. B. 87, 211 (2014).

16. A.A. Kistanov, S.V. Dmitriev. Letters on Materials. 2, 143 (2012).

17. V.I. Dubinko, P.A. Selyshchev, J.F.R. Archilla, Phys. Rev. E 83, 041124 (2011).

18. M.G. Velarde, J. Comput. Appl. Math. 233, 1432 (2010).

19. M.G. Velarde, W. Ebeling, A.P. Chetverikov. Eur. Phys. J. B 85, 291 (2012).

20. A.P. Chetverikov, W. Ebeling, M.G. Velarde. Eur. Phys. J. ST 222, 2531 (2013).

21. M.O. Sales, F.A.B.F. de Moura. J. Phys.: Condens. Matter 26, 415401 (2014).

22. M.E. Manley, A.J. Sievers, J.W. Lynn, S.A. Kiselev, N.I. Agladze, Y. Chen, A. Llobet, A. Alatas. Phys. Rev. B. 79, 134304 (2009).

23. A.A. Kistanov, R.T. Murzaev, S.V. Dmitriev, V.I. Dubinko, V.V. Khizhnyakov. JETP Lett. 99, 353 (2014).

24. S.V. Dmitriev, A.A. Kistanov, V.I. Dubinko. Springer Series in Materials Science. 221, 205 (2015).

25. E.A. Korznikova, S.Y. Fomin, E.G. Soboleva, S.V. Dmitriev. JETP Lett. 103, 277 (2016).

26. S. Timoshenko, J.N. Goodier. Theory of elasticity. McGraw-Hill, New York (1951). 